\title{
Recent Advances in AI based Automated Personalized Nutrition System: Future Need of Healthcare
}

\author{
Dharmendra Sharma ${ }^{1}$, Sachin Patel $^{2}$
}

${ }^{1}$ Narsee Monjee Institute of Management Studies (NMIMS University, Indore), India, dr.dksharma8@ gmail.com

${ }^{2}$ SIRT, SAGE University Indore, India, er.sachinpcst@gmail.com

\begin{abstract}
Intake of healthy and balanced nutrition is the most important and common need of human being to develop strong immune system against the life-threatening diseases such as cancer, diabetic, cardiovascular diseases and covid-19. Universal diet for each individual is not a biological correct statement hence there is a future need of society to have an automated low-cost personalized diet system. From last decade, due to technical advancement in artificial intelligence, social and mobile network it is very easy to share food images, recipes and food diaries on common platform which will be further be helpful to develop large-scale data. Due to this technical development there is lot of research scope for significant area of research known as food computing and energy expenditure calculations. In this research paper, we have provided compressive overview of different emerging innovations in the field of food classification and energy expenditure calculations. Comparative analysis of each method is provided in tabular form to get more insight to each application. This paper is concluded with findings and future challenges in the field of food computing and energy expenditure calculations. It is the future need to shape this area by overcoming challenges to provide economical and personalized nutrition system to the society.
\end{abstract}

Key words: Computer vision, Deep learning, Energy expenditure, Food classification system, Machine learning, Sensor technology, and Personalized nutrition system.

\section{INTRODUCTION}

In the modern world, due to change in lifestyle and increase in work burden many people are facing problem to maintain the healthy diet and due to this there is a significant increase in different chronic diseases. Due to this, there is a constant demand to have automated nutrition system in the market. There are many nutrition systems available in the market which works on universal diet, but universal diet system is not suitable for each and every common man on the earth [1]. Available personalized nutrition system is labour concentrated hence it is not economical. Due to the technical advancement in sensor technology, wearable device and artificial intelligence there is a positive hope to have a personalized nutrition system to conquer the drawbacks of long-established food tracking system which is less accurate and labor concentrated [2] Personalized nutrition system is the integration of various complicated factors such as food classification and nutrition calculation system, energy expenditure calculations system and personalized metabolism and physiological health [3] which is illustrated in figure-1.

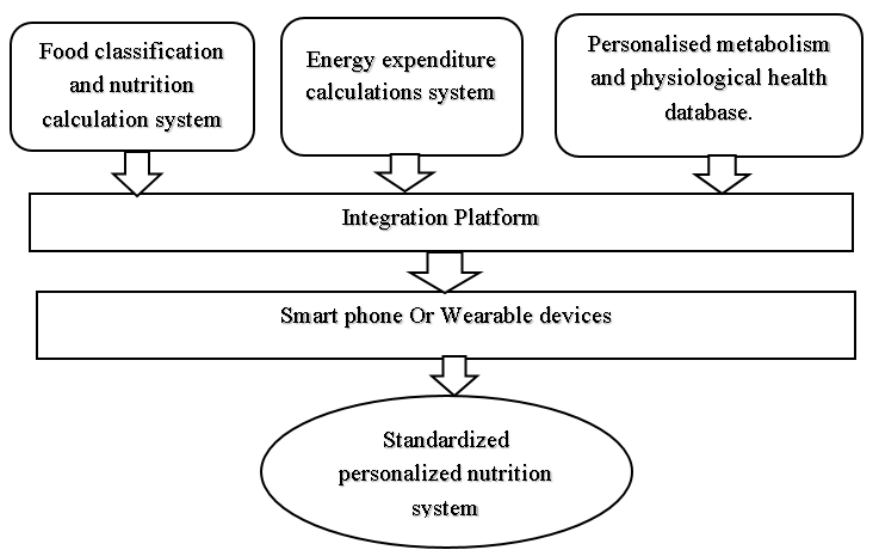

Figure-1: Illustration of standardized personalized nutrition framework

Food classification and nutrition calculation block basically uses machine learning or deep learning approach to extract different features from food images. It is further used for nutrition calculations while the energy expenditure calculation uses sensor technology to measure the human activity in free-living conditions and with this calculation it is important to understand the personalized metabolism of each user to suggest the suitable diet on individual basis. We have kept this personalized nutrition framework in mind to perform literature survey from different databases such as Science direct, IEEE explore and Springer. For better understanding we have divided the paper hereafter into three different section, section-2: describes the technical advancement in Food classification and nutrition calculations systems. While section- 3: gives an overview of recent development in energy expenditure system section-4: Highlights the findings and future challenges in this research area and finally paper conclude with section-5. 


\section{TECHNICAL ADVANCEMENT IN FOOD CLASSIFICATION AND NUTRITION CALCULATIONS SYSTEMS}

This section will provide survey of different innovative techniques used for food classification and nutrition calculations. As shown in figure-2, food classification technique takes the food image as an input dataset and with the help of computer vision algorithms or by deep learning approach food dataset is classified further for nutrition calculations.

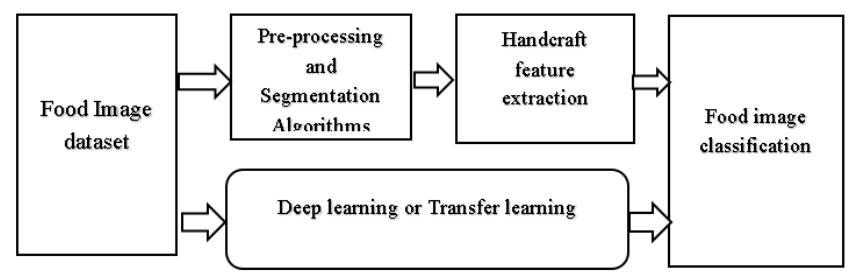

Figure-2: Generalized research approach for food image classification

As per literature survey, food classification approach is divided according to the feature extraction techniques as a hand- crafted features extraction and learned features extraction.

\subsection{Hand-crafted features Extraction}

Food images are complex dataset for classification hence only single feature is not sufficient for food recognition and quantity estimation hence there is need to extract maximum number of features with the help of computer vision algorithms and different features extraction techniques. These features are varying from local texture properties, color information, local binary patterns, Entropy-Based Categorization, Fractal Dimension Estimation and Gabor Based Image Decomposition. These features are further given to SVM and KNN for classification.

E Silva et al. [4] uses interactive mobile based model in which automated mobile based food recognition system provides an assessment of food images and tracks the users dietary and physical activity with additional one unique extra feature which is real-time energy balance monitoring by use of machine learning and computer vision algorithm. In this system food recognition is done with smartphone which take pictures of meal at four different angles and then ostu thresholding was performed to segment background and foreground. After segmentation Local Binary Patterns (LBP), color information, texture and Scale-Invariant Feature Transform (SIFT) features are extracted and finally images are classified with SVM and KNN on 60 types of dataset and each dataset consists of 1000 images. In this paper, the claimed machine leaning model accuracy of $65.5 \%$. Parisa Pouladzadeh et al. [5] proposed another system for Measuring Calorie and Nutrition from food Images. In this system photos of images are pre-processed and segmented with the help of image transformation and contour recognition and finally Gabor filter is used to measure local texture properties in frequency domain. With the help of Gabor filter estimate further calculation of mean and standard deviation of the energy was performed. In this system, the author calculated four types of features like color, shape, size and texture and given to SVM for classification with 10 cross folding concepts. Classification accuracy varies from $86 \%$ for food images while it recorded highest $92.21 \%$ for fruit images. Classification accuracy can be enhanced with the help of better segmentation techniques. Oliveira, L et al. [6] proposed novel camera equipped mobile application for identification and classification of food images. In this application user has to click the picture of the image and select the initial seed for segmentation of food image by dynamic region growing algorithms which run over multiple feature space and then this feature is provided for SVM for final classification which was performed on 1500 training and 2300 testing images.

Ye He et.al [7] proposed a dietary assessment system which will keep track records of daily food intake taken in a meal with the help of segmentation feature extraction and weight calculation. In this proposed system, Graph based image segmentation method was used to segment the image and internal difference of a segmented region is defined to be the largest weight in the spanning tree. For feature extraction, three texture descriptors are extracted which are partial Dependence Matrix (GOSDM), Entropy-Based Categorization and Fractal Dimension Estimation (EFD) and Gabor Based Image Decomposition and Fractal Dimension Estimation (GFD) are used. This method was tested on 1453 food images and obtained classification accuracy of $63 \%$. Weight estimation was done on the basis of area-based calculations. Comparative analysis of all the surveyed applications are provided in table-1.

Table-1.: Comparative analysis of Hand-crafted feature techniques.

\begin{tabular}{|c|c|c|c|c|}
\hline Authors & $\begin{array}{l}\text { Hand-crafted feature } \\
\text { extraction }\end{array}$ & $\begin{array}{l}\text { Classification } \\
\text { Algorithm }\end{array}$ & Dataset & $\begin{array}{l}\text { Classification } \\
\text { accuracy. }\end{array}$ \\
\hline $\begin{array}{l}\text { E Silva et } \\
\text { al. [4] }\end{array}$ & $\begin{array}{l}\text { Local Binary Patterns, } \\
\text { texture and Scale- } \\
\text { Invariant Feature } \\
\text { Transform (SIFT) }\end{array}$ & SVM and KNN & $\begin{array}{l}60 \text { types of } \\
\text { dataset each } \\
\text { set consists } \\
1000 \text { images. }\end{array}$ & $65.5 \%$ \\
\hline $\begin{array}{l}\text { Parisa et } \\
\text { al. [5] }\end{array}$ & $\begin{array}{l}\text { Local texture properties } \\
\text { in frequency domain }\end{array}$ & SVM & $\begin{array}{l}\text { Testing is } \\
\text { done on user } \\
\text { dataset. }\end{array}$ & $\begin{array}{l}86 \% \text { for food } \\
\text { images and } \\
92.21 \% \text { for } \\
\text { Fruit images. }\end{array}$ \\
\hline $\begin{array}{l}\text { Oliveira et } \\
\text { al. [6] }\end{array}$ & $\begin{array}{l}\text { Statistical feature } \\
\text { extraction }\end{array}$ & SVM & $\begin{array}{l}3800 \text { food } \\
\text { images. }\end{array}$ & $87 \%$ \\
\hline $\begin{array}{l}\text { Ye He et al. } \\
{[7]}\end{array}$ & $\begin{array}{l}\text { Partial Dependence } \\
\text { Matrix, Entropy-Based } \\
\text { Categorization, and } \\
\text { Fractal Dimension } \\
\text { Estimation }\end{array}$ & KNN & $\begin{array}{l}1453 \text { food } \\
\text { images }\end{array}$ & $63 \%$ \\
\hline
\end{tabular}

In most of the research application the depth of food images is considered uniform which is one of the causes for reduction in classification accuracy. As food image is a complex image which needs to calculate a greater number of features and improvement in dimension reduction algorithm is the need of 
research in this area and also very less articles on this concept indicate research direction is diverted towards deep learning or transfer learning.

\subsection{LEARNED FEATURES EXTRACTIONS}

Due to the availability of big data, CNN acts as a central point of attraction for image recognition, and image retrieval. CNN is the combination of many linear and non-linear regions operators. These operators are designed to learn the features in end-to-end manners. During this literature survey, we come across different new and pre-trained CNN algorithms which was used for food recognition task.

Sundaravadivel et al. [8] proposed novel IOT based system known as Smart-log which consists of a 5-layer deep leaning model which is closely connected to hidden layers in order to measure nutritional balance after each meal .Another algorithm is based on Bayesian networks to determine nutrient features from food materials and also suggest meal or related recipes. This algorithm is the combination of several Bayesian classifiers. The proposed IOT system is fully automated and act as a first solution which uses Bayesian algorithms with combination of 5-layer perceptron neural network for diet monitoring with classification accuracy of 98.6\% and this system is designed for child care household application, but with certain modifications it can be used for automatic prediction of Adult diet. This system can be integrated with physiological monitoring to keep track record of the physical activity. This system is cost effective as well proven as a highly accurate.

$\mathrm{CNN}$ is the most powerful tool to learn features in more robust and expressive way. Ciocca, $\mathrm{G}$ et al. [9] proposed CNN based residual network with 50 layers architecture to extract features from the food- 475 database which was developed and available publically and provide satisfactory results and act as one step forward to tackle challenges in food recognition Generally, Google Net and ResNet requires larger number of parameters in food recognition which will increase computational complexity to overcome this Jahani Heravi, et al.[10] proposed 23-layer architecture which is comparable to both deep learning architecture and obtained accuracy of 99.14\% on Food 101and 96.63 on UECFood-256 datasets with a smaller number of parameters.

In order to classify good and bad quality beer, Dębska, B et al. [11] proposed ANN classifier with the help of 12 pre-defined features such as percentage of alcohol, $\mathrm{pH}$, and percentage of $\mathrm{CO} 2$ etc.). This classification is performed on two subsets such as good and bad quality beers and found classification accuracy of $100 \%$. Transfer learning is having advantages to use pretrained models instead of designing from the scratch on this concept Rajayogi J R et al. [12] performed transfer learning with the help of IncceptionV3, VGG16, VGG19 and ResNet to classify Indian 20 food dataset in which each dataset consists 500 images. Results analysis shows that Google InceptionV3 performed better as compared to other deep learning approach. In this research, results are hampered due to noise in the image and a smaller number of datasets. Results of Google InceptionV3 was recorded 87.9\%. $\mathrm{S}$.

Mezgec et al. [13] developed novel deep learning architecture known as NutriNet to classify the fake food image which is based on pixel-level classification. This system was tested on 225,953 datasets with classification accuracy of $86.72 \%$. Author has used FCN-8s to train the model and can act as a baseline model for future studies. This was first approach of joint segmentation and pre-processing of image by deep learning.

G. Ciocca[14] proposed another novel approach for dietary monitoring applications in which each image act as real canteen tray and each tray consists multiple instances of food classes. This application consists of total 1027 canteen tray for total 3616 food instances which belongs to 73 food classes. These food images are segmented carefully with the help of polygonal boundaries and obtained classification accuracy of 79\% with convolutional neural network. Comparative analysis of all the surveyed article is given in Table-2.

Table-2: Comparative analysis of learned features techniques.

\begin{tabular}{|c|c|c|c|}
\hline Author & Deep learning Algorithm & Dataset/additional feature & $\begin{array}{c}\text { Classification } \\
\text { accuracy }\end{array}$ \\
\hline $\begin{array}{l}\text { Sundaravadivel } \\
\text { et al. [8] }\end{array}$ & $\begin{array}{l}\text { 5-layer deep leaning } \\
\text { model with Bayesian } \\
\text { classifiers }\end{array}$ & $\begin{array}{l}\text { Can be integrated with activity } \\
\text { monitoring, highly accurate } \\
\text { and cost effective }\end{array}$ & $98.6 \%$ \\
\hline $\begin{array}{l}\text { Ciocca, Getal. } \\
{[9]}\end{array}$ & $\begin{array}{l}\text { CNN based residual } \\
\text { network with } 50 \text { layers } \\
\text { architecture }\end{array}$ & Food-475 database & $78 \%$ \\
\hline $\begin{array}{l}\text { Jahani Heravi, } \\
\text { et al. [10] }\end{array}$ & $\begin{array}{l}\text { 23-layer architecture CNNN } \\
\text { architecture }\end{array}$ & $\begin{array}{c}\text { Food } 101 \text { and UECFood-256 } \\
\text { dataset and comparable to } \\
\text { Google Net and ResNet. }\end{array}$ & $99.14 \%$ and $96.63 \%$ \\
\hline $\begin{array}{l}\text { Dębska, B et al. } \\
\text { [11] }\end{array}$ & $\begin{array}{l}\text { ANN classifier with } 12 \\
\text { predefined features }\end{array}$ & $\begin{array}{l}\text { To classify two subsets of } \\
\text { good and bad quality of beer. }\end{array}$ & $\begin{array}{l}100 \% \text { for two class } \\
\text { classification. }\end{array}$ \\
\hline $\begin{array}{l}\text { Rajayogi JR et } \\
\text { al. [12] }\end{array}$ & $\begin{array}{l}\text { Performed transfer } \\
\text { learning with help of } \\
\text { IncceptionV3, VGG16, } \\
\text { VGG19 and ResNet }\end{array}$ & $\begin{array}{l}\text { Indian } 20 \text { food dataset each } \\
\text { consist } 500 \text { images }\end{array}$ & $\begin{array}{l}\text { Google InceptionV3 } \\
\text { performed better with } \\
\text { classification } \\
\text { accuracy of } 87.9 \%\end{array}$ \\
\hline $\begin{array}{l}\text { S. Mezgec et al. } \\
{[13]}\end{array}$ & $\begin{array}{l}\text { NutriNet, deep learning } \\
\text { model }\end{array}$ & $\begin{array}{c}\text { Tested on } 225,953 \text { image } \\
\text { datasets to classify good and } \\
\text { bad quality food. }\end{array}$ & $86.72 \%$ \\
\hline $\begin{array}{l}\text { G. Ciocca et al. } \\
{[14]}\end{array}$ & CNN Architecture. & Tested on 3616 food instances & $79 \%$ \\
\hline
\end{tabular}

In order to classify food images different authors have designed new deep learning architectures in which layers of architecture are variable from 5 to 50 and tested on different datasets while some authors have used the concept of transfer learning with the support of existing architectures such as IncceptionV3, VGG16, VGG19 and ResNet. Classification accuracy variation was observed from $79 \%$ to the $98.6 \%$. Deep learning model performs on optimal level when there is a two-class classification, but in food classification accuracy variations is larger one and hence indicates that still there is a research scope for improvement. Sharma and Jain [15 - 16] develop a weighting based model to classify the food by changing the context at runtime. 


\section{RECENT DEVELOPMENT IN ENERGY EXPENDITURE SYSTEM}

This section will describe recent development of estimation of caloric expenditure of human body during the daily activity. Measurement of physical activity is most complicated and the variable component to access during free-living condition. As per research articles, most precise method for energy measurement is doubly-labelled water (DLW) assessment with combination of measurement of the resting metabolic rate (RMR) with the help of calorimetry but this method requires high grade technical experts hence expensive [17-18]. Different researchers have proposed various sensors and integration of this sensors with diet system which will be helpful to design more accurately personalized nutrition system. Basic building block of this system is different sensor technology, microcontroller, wearable device or IOT which is shown in figure 3.

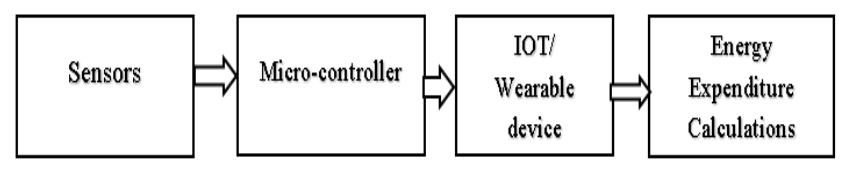

Figure-3: Generalized approach for energy Expenditure calculation

Ling Xiao et al. [19] proposed kinetic energy harvesting $(\mathrm{KEH})$ device which is an alternative to the accelerometer and output voltage of $\mathrm{KEH}$ is used to classify the activity intensity of seven different activities in free-living conditions. Experimental results of this approach provided an accuracy of $95.7 \%$. This validation provides effectiveness of $\mathrm{KEH}$ device over accelerometer with energy saving option.

Pande et al. [20] proposed accelerometer and barometer sensor to measure energy expenditure during walking, standing, and climbing upstairs or down stairs with integration with mobile device. In order to develop generic regression model machine learning and artificial intelligence algorithm was used and found correlation of $89 \%$. The results of this algorithm were found superior than Fitbit and Nike+ Fuel Band. These results are calibrated against calorimeter readings.

Gardner et al. [21] proposed hand-held and low-cost calorimeter for the measurement of energy expenditure from human breath. In order to calculate energy burned by the body over the given time period they used prototype device which is the combination of oxygen and carbon dioxide sensors and compared with the commercial sensor which recorded an error of $7.9 \%$ in controlled environments. Further modifications are required to be done to enable it to work outside the hospital environment then this tool can act as a biomarker in exhaled breath for diagnosis of different diseases.

P. Russo et al. [22] proposed electromagnetic contactless motion sensor in which velocity and kinetic energy associated with moving target are calculated with the help of elaboration of spectrogram intensity and validated with human object moving arm and provided very promising results.

In case of post-stroke patients with partial loss of neurological function it is very important to estimate the energy expenditure to understand the status of physical recovery.

J. Zhang et al. [23] proposed homemade micro thermal flow meter which is used to detect motion velocity and Kalman based algorithm is used to extract motion acceleration from flow sensor output. This method is free of accumulation error and suitable rehabilitation training. Wearable sensors used for prediction of energy expenditure at free-living condition is the most complex task due to sensor placement, user agreement and validation methods.

In order to validate single ear-worn accelerometer, L. Bouarfa et al. [24] developed prediction model which consists of 10 different physical activity and found tenfold cross validation error of 0.24 and correlation of .074 .

In order to perform objective assessment of energy expenditure during indoor workout intelligent video processing concept used by Y. Yang et al. [25] which is based on smart camera which is capable to measure repetition and intensity level and used algorithm to analyze body movement and compared with the gold standard indirect calorimetry apparatus. Most of the research articles confirm the effectiveness of accelerometer-based energy expenditure calculation but to overcome shortcomings of accuracy of existing algorithm, W. Wang et al. [26] proposed stoop activity energy expenditure with the help of novel transducer and obtained accuracy of $84 \%$.

In order to overcome life-style related chronic diseases, D. Ravì et al. [27] proposed real-time food recognition system which is based on the daily activity and energy expenditure estimation. Daily energy expenditure is achieved by mobile based built-in inertial motion sensors and food recognition computer vision algorithm. This system is validated with the state-of-art method and found improved accuracy. Novel approach of deep convolutional neural network features is utilized for energy expenditure calculation by B. Wang et al. [28] in this system, validated accuracy was around $82.3 \%$ shows importance of deep learning approach for activity monitoring.

Fusion of visual and accelerometer sensor was proposed by L. Tao et al. [29] individual-independent framework for energy expenditure in controlled environment. Validation results shows reduction in errors by $8 \%$ in only visual and $18 \%$ in only accelerometer conditions. Energy consumption in the body is directly proportional to the oxygen consumption hence T. Nagata et al. [30] proposed oxygen consumption estimation with the support of 6-axis motion sensor which is the combination of 3-axis accelerometer and 3-axis gyroscope 
and validated results of this method with machine learning algorithm. Comparative analysis of all the reviewed articles are shown in table-3.

Table-3: Comparative analysis of energy expenditure system.

\begin{tabular}{|c|c|c|}
\hline Author & Sensor technology & Significant features if any \\
\hline $\begin{array}{l}\text { Ling Xiao et } \\
\text { al. [19] }\end{array}$ & $\begin{array}{l}\text { Kinetic energy harvesting } \\
(\mathrm{KEH}) \text { device. }\end{array}$ & $\begin{array}{l}\text { Which act as an alternative to } \\
\text { accelerometer. } \\
\text { Measures seven different } \\
\text { activity with an accuracy of } \\
95.7 \% \text {. }\end{array}$ \\
\hline $\begin{array}{l}\text { Pande et al. } \\
{[20]}\end{array}$ & $\begin{array}{l}\text { Accelerometer and barometer } \\
\text { sensor }\end{array}$ & $\begin{array}{l}\text { Measures energy expenditure } \\
\text { during walking, standing, and } \\
\text { climbing upstairs or down stairs. } \\
\text { Validated with machine learning } \\
\text { algorithm and found accuracy of } \\
89 \% \text {. }\end{array}$ \\
\hline $\begin{array}{l}\text { Gardner et al. } \\
\text { [21] }\end{array}$ & $\begin{array}{l}\text { Combination of oxygen and } \\
\text { carbon } \\
\text { electrochemical sensors. }\end{array}$ & $\begin{array}{l}\text { Validated with commercial } \\
\text { sensors and recorded accuracy of } \\
91.9 \% \text {. }\end{array}$ \\
\hline $\begin{array}{l}\text { P. Russo et al. } \\
\text { [22] }\end{array}$ & $\begin{array}{l}\text { Electromagnetic contactless } \\
\text { motion sensor. }\end{array}$ & $\begin{array}{l}\text { Use of spectrogram intensity and } \\
\text { validated with human object } \\
\text { moving arm and obtained } \\
\text { satisfactory results. }\end{array}$ \\
\hline $\begin{array}{l}\text { J. Zhang et al. } \\
\text { [23] }\end{array}$ & Micro thermal flow meter & $\begin{array}{l}\text { This method is free of } \\
\text { accumulation error and suitable } \\
\text { rehabilitation training. }\end{array}$ \\
\hline $\begin{array}{l}\text { L. Bouarfa et } \\
\text { al. [24] }\end{array}$ & Single ear-worn accelerometer & $\begin{array}{l}\text { Tested with } 10 \text { different phy sical } \\
\text { activity and found tenfold cross } \\
\text { validation error of } 0.24 \text { and } \\
\text { correlation of } 074 \text {. }\end{array}$ \\
\hline $\begin{array}{l}\text { Y. Yang et al. } \\
\text { [25] }\end{array}$ & $\begin{array}{l}\text { Intelligent video processing } \\
\text { with smart camera. }\end{array}$ & $\begin{array}{l}\text { This system is capable to } \\
\text { measure repetition and intensity } \\
\text { level of human body and } \\
\text { compared with gold standard } \\
\text { indirect calorimeter apparatus. }\end{array}$ \\
\hline $\begin{array}{l}\text { W. Wang et al. } \\
\text { [26] }\end{array}$ & $\begin{array}{l}\text { Stoop activity } \\
\text { energy } \\
\text { expenditure with help of } \\
\text { transducer. }\end{array}$ & $\begin{array}{l}\text { It acts as an alternative to } \\
\text { Accelerometer with accuracy of } \\
84 \%\end{array}$ \\
\hline $\begin{array}{l}\text { D. Ravi et al. } \\
\text { [27] }\end{array}$ & $\begin{array}{l}\text { Built-in inertial motion sensors } \\
\text { and food recognition computer } \\
\text { vision algorithm. }\end{array}$ & $\begin{array}{l}\text { Integrated mobile based } \\
\text { nutrition system which combine } \\
\text { activity measurement and food } \\
\text { classification system. }\end{array}$ \\
\hline
\end{tabular}

\section{FINDINGS AND FUTURE CHALLENGES}

During this literature survey, we come across different innovative nutrition and energy expenditure systems. During this study we have recorded some important findings which will be helpful to decide the future path of research in this area. In food classification and nutrition calculations, two approaches are utilized by different authors which are either hand-crafted features extraction or learned features extraction. In hand-crafted features extraction computer vision algorithms are used for segmentation of food image and then different feature extraction techniques are used which are local binary patterns, Partial dependence matrix, entropy-based feature etc. and finally classification is done with SVM or KNN machine learning algorithms. As food is the most complex object to classify hence accuracy variation takes place from $63 \%$ to $92 \%$. In this approach more refine version of computer vision algorithms and feature extraction techniques are required to be developed to reach all the measurements on optimal levels [31].

Syamla and Nalini[32] worked on extraction of impartent aspect by using different machine learning alogithms the compare positive and negative opinion by defining an aspect based framework. .Manoj and sasikanth[33] analyze the different risk factors of data science with Bigdata. They highlighted the risk of association of client with online content in online networking.

In learned feature approach, Sharma and Jain developed a similarity measurement model [34]. Some authors try to design deep learning algorithms in terms of different numbers of hidden layers which varies from 5-23 to extract features from the food images while others used concepts of transfer learning.

Energy expenditure calculations of human body in free-living condition is another complex task. During this study, we come across different innovative techniques in sensor technology which varies from accelerometer, thermal flow meter, electromagnetic contactless motion sensor, intelligent video processing and fusion of visual and accelerometer. For the validation some authors used the concept of machine and deep learning to get more accurate results.

This study identifies certain challenges in this field which need to be tackled by future researcher to design more accurate and cost-effective nutrition management system for welfare of society.

1. Vast food diversity around the world which will make difficult to design versatile food classification system hence there is a need to make continuous effort to scale up the food image database.

2. Validation of food classification system is performed on different dataset hence comparison on common platform is very difficult and hence need to develop a gold standard for validation of all the system on common platform.

3. Food is a complex image dataset hence there is a need of development of more advanced version of computer vision algorithms for segmentation of food images.

4. More advanced version of deep learning algorithms is needed to enhance the classification accuracy.

5. Even though there are many sensors developed for energy expenditure calculations, but still accuracy is the major concern for sensor design and hence there is a need of more accurate, portable sensors which can be easily integrated with wearable device.

6. Also, there is a need of integration of Individual Metabolism and physiological health database with energy expenditure and food classification and nutrition calculations system.

For the design of accurate and cost-effective personalized nutrition management system there is a need to address all above challenges in future.

\section{CONCLUSION}

Personalized nutrition management system is the future need of society due to change in life style and exposure to more chronic diseases. This system should be the combination of 
food classification, nutrition calculations and energy expenditure calculations. In this study, we have come across different individual approaches which should be integrated together in future to develop more reliable personalized nutrition management system. To achieve this research objective, there is a need of more refine version of computer vision and artificial intelligence algorithms with support of portable and accurate sensor technology and larger dataset for ground truth validation. In future, these modifications surely will be helpful to design more reliable and cost-effective nutrition system for healthcare.

\section{REFERENCES}

1. Resende Silva, B. V., \& Cui, J. "A Survey on Automated Food Monitoring and Dietary Management Systems". Journal of Health \& Medical Informatics, 08(03), 2017

.https://doi.org/10.4172/2157-7420.1000272.

2. Resende e Silva, B. V., Rad, M. G., Cui, J., McCabe, M., \& Pan, K. "A Mobile-Based Diet Monitoring System for Obesity Management". Journal of Health \& Medical Informatics, 2018. https://doi.org/10.4172/2157-7420.1000307.

3. Pawar, S., \& Sharma, K. K. "Refining healthcare in terms of diabetic care: Future area of scope for artificial intelligence". International Journal of Recent Technology and Engineering, 8(2 Special Issue 8), 888-893,2019.

https://doi.org/10.35940/ijrte.B1003.0882S819.

4. Resende e Silva, B. V., Rad, M. G., Cui, J., McCabe, M., \& Pan, K. "A Mobile-Based Diet Monitoring System for Obesity Management". Journal of Health \& Medical Informatics,09(02),2018. https://doi.org/10.4172/2157-7420.1000307.

5. Sundaravadivel, P., Kesavan, K., Kesavan, L., Mohanty, S. P., \& Kougianos, "E Smart-Log: A Deep-Learning Based Automated Nutrition Monitoring System in the IoT'. IEEE Transactions on Consumer Electronics, 64(3), 390-398,2018. https://doi.org/10.1109/TCE.2018.2867802.

6. Oliveira, L., Costa, V., Neves, G., Oliveira, T., Jorge, E., \& Lizarraga, M. . “A mobile, lightweight, poll-based food identification system". Pattern Recognition, 47(5), $1941-1952,2014$ https://doi.org/10.1016/j.patcog.2013.12.006.

7. He, Y., Xu, C., Khanna, N., Boushey, C. J., \& Delp, E. J. "Food image analysis: Segmentation, identification and weight estimation". In Proceedings - IEEE International Conference on Multimedia and Expo. 2013. https://doi.org/10.1109/ICME.2013.6607548.

8. S. Mezgec and B. K. Seljak, "Using Deep Learning for Food and Beverage Image Recognition," IEEE International Conference on Big Data (Big Data), Los Angeles, CA, USA, 2019, pp. 5149-5151, 2019. doi: 10.1109/BigData47090.2019.9006181.
9. Ciocca, G., Napoletano, P., \& Schettini, R. “CNN-based features for retrieval and classification of food images" A R T I C L E I N F O Communicated by Nikos Paragios. Computer Vision and Image Understanding, 176-177, 70-77, 2018.

https://doi.org/10.1016/j.cviu.2018.09.001.

10. Jahani Heravi, E., Habibi Aghdam, H., \& Puig, D. “An optimized convolutional neural network with bottleneck and spatial pyramid pooling layers for classification of foods". Pattern Recognition Letters, 105, 50-58, 2018. doi:10.1016/j.patrec.2017.12.007

11. Debska, B., \& Guzowska-Świder, B. "Application of artificial neural network in food classification". Analytica Chimica Acta, 705(1-2), 283-291, 2011. https://doi.org/10.1016/j.aca.2011.06.033.

12. Rajayogi, J. R., Manjunath, G., \& Shobha, G.. "Indian Food Image Classification with Transfer Learning", In CSITSS 2019 - 2019 4th International Conference on Computational Systems and Information Technology for Sustainable Solution, Proceedings. Institute of Electrical and Electronics Engineers Inc. 2019. https://doi.org/10.1109/CSITSS47250.2019.9031051.

13. Mezgec, S., \& Koroušić Seljak, B. "NutriNet: A Deep Learning Food and Drink Image Recognition System for Dietary Assessment. Nutrients", 9(7), 657, 2017. doi:10.3390/nu9070657.

14. G. Ciocca, P. Napoletano and R. Schettini, "Food Recognition: A New Dataset, Experiments, and Results," in IEEE Journal of Biomedical and Health Informatics, vol. 21, no. 3, pp. 588-598, May 2017, doi: 10.1109/JBHI.2016.2636441.

15. D.sharma, s. Jain, "Content sharing in information storage and retrieval system using tree representation of documents", Proceedings of the 2014 Conference on IT in Business, Industry and Government: An International Conference by CSI on Big Data, CSIBIG 2014.

16. D.Sharma, S.Jain, "Context-based weighting for vector space model to evaluate the relation between concept and context in information storage and retrieval system”, IEEE International Conference on Computer Communication and Control, IC4 2015.

17. Klein, P. D., James, W. P., Wong, W. W., Irving, C. S., Murgatroyd, P. R., Cabrera, M., ... Nichols, B. L. "Calorimetric validation of the doubly-labelled water method for determination of energy expenditure in man". Human Nutrition. Clinical Nutrition, 38(2), 95-106, 1984.

18. Seale, J. L., Conway, J. M., \& Canary, J. J. "Seven-day validation of doubly labeled water method using indirect room calorimetry". Journal of Applied Physiology, 74(1), 402-409, 1993. https://doi.org/10.1152/jappl.1993.74.1.402.

19. Xiao, L., Wu, K., Tian, X., \& Luo, J. "Activity-specific caloric expenditure estimation from kinetic energy harvesting in wearable devices. Pervasive and Mobile 
Computing', $\quad 101185, \quad 2020$. https://doi.org/10.1016/j.pmcj.2020.101185.

20. Pande, A., Zeng, Y., Das, A. K., Mohapatra, P., Miyamoto, S., Seto, E., Han, J. J. "Energy expenditure estimation with smartphone body sensors". In BODYNETS 2013 - 8th International Conference on Body Area Networks (pp. 8-14). ICST, 2013. https://doi.org/10.4108/icst.bodynets.2013.253699.

21. J. W. Gardner and T. A. Vincent, "Towards point of care human energy expenditure measurement on a hand-held breath analyser," ISOCS/IEEE International Symposium on Olfaction and Electronic Nose (ISOEN), Montreal, QC, 2017, pp. 1-3, 2017. doi: 10.1109/ISOEN.2017.7968901.

22. P. Russo, V. Di Mattia, G. Manfredi, A. De Leo and G. Cerri, "Radar Doppler technique to estimate the energy expenditure of human physical activities," 12th European Conference on Antennas and Propagation (EuCAP 2018), London, pp. 1-5, 2018. doi: 10.1049/cp.2018.0977.

23. J. Zhang, S. Liu and R. Zhu, Motion Velocity, "Acceleration and Energy Expenditure Estimation Using Micro Flow Sensor," in IEEE Access, vol. 7, pp. 75901-75909, 2019. doi: 10.1109/ACCESS.2019.2921978.

24. L. Bouarfa, L. Atallah, R. M. Kwasnicki, C. Pettitt, G. Frost and G. Yang, "Predicting Free-Living Energy Expenditure Using a Miniaturized Ear-Worn Sensor: An Evaluation Against Doubly Labeled Water," in IEEE Transactions on Biomedical Engineering, vol. 61, no. 2, pp. 566-575, Feb. 2014. doi: 10.1109/TBME.2013.2284069.

25. Yang, Y., Liu, C., Tsow, F., Shao, D., Yu, H., Xia, S., \& Tao, N. "Remote Quantification of Workout Energy Expenditure with a Cell Phone Camera". IEEE Sensors Journal, 16(23), 8263-8270, 2016. https://doi.org/10.1109/JSEN.2016.2519906.

26. W. Wang, W. Wei, Z. Qu, L. Cheng, J. Gu and X. Lin, "Research on Human Stoop Activity Energy Expenditure Detection Algorithm Based on AHRS Transducer," 2019 IEEE International Conference on Mechatronics and Automation (ICMA), Tianjin, China, pp. 916-920,2019.

doi: 10.1109/ICMA.2019.8816313.

27. D. Ravì, B. Lo and G. Yang, "Real-time food intake classification and energy expenditure estimation on a mobile device," 2015 IEEE 12th International Conference on Wearable and Implantable Body Sensor Networks (BSN), Cambridge, MA, , pp. 1-6, 2015 doi: 10.1109/BSN.2015.7299410.

28. B. Wang, L. Tao, T. Burghardt and M. Mirmehdi, "Calorific Expenditure Estimation Using Deep Convolutional Network Features," 2018 IEEE Winter Applications of Computer Vision Workshops (WACVW), Lake Tahoe, NV, pp. 69-76, 2018.

doi: 10.1109/WACVW.2018.00014.
29. L. Tao et al., "Energy expenditure estimation using visual and inertial sensors," in IET Computer Vision, vol. 12 , no. 1, pp. 36-47, 2 2018, doi: 10.1049/iet-cvi.2017.0112.

30. T. Nagata et al., "VO2 estimation using 6-axis motion sensor with sports activity classification," 2016 38th Annual International Conference of the IEEE Engineering in Medicine and Biology Society (EMBC), Orlando, FL, pp. 4735-4738, 2016. doi: 10.1109/EMBC.2016.7591785.

31. Sharma, K. Kr., Pawar, S. D., \& Bali, B. 'Proactive Preventive and Evidence-Based Artificial Intelligence Models: Future Healthcare" (pp. 463-472), https://doi.org/10.1007/978-981-15-0633-8_44.

32. Syamala, Nalini, "A Deep Analysis on Aspect based Sentiment Text Classification Approaches", International Journal of Advanced Trends in Computer Science and Engineering, Volume 8, No.5, September October 2019.

33. B.Manoj, .Sasikanth, "Analysis of Data Science with the use of Big Data", International Journal of Advanced Trends in Computer Science and Engineering, Volume 7, No.6, November - December 2018

34. D.Sharma, S. Jain, "A model for context-based similarity measurement of opinions by using dynamic weighting scheme ", International Journal of Emerging Trends in Engineering Research, 2020. 